\title{
¿Qué hay en un nombre?
}

\section{What's in a name?}

\author{
Eugenio Matijasevic • Bogotá D.C. (Colombia)
}

En una vieja canción de Mecano (en este caso vieja quiere decir, ni más ni menos, que ninguno de mis alumnos la conoce) se oye como estribillo: "quise cortar la flor más tierna del rosal/ pensando que de amor no me podría pinchar/ y mientras me pinchaba me enseñó una cosa:/ que una rosa es una rosa es una rosa" (1).

La frase final del estribillo fue tomada y traducida de Sacred Emily, un poema de Gertrude Stein publicado en 1913 en el que declara sin ambages: "Rose is a rose is a rose is a rose" (Rosa es una rosa es una rosa es una rosa) (2), dando desde la poesía una clara muestra de que conoce a profundidad los principios de la lógica clásica. Los tres principios fundamentales del razonamiento lógico en el pensamiento clásico y medieval (pero, sin lugar a dudas, útiles también en el pensamiento contemporáneo) son el principio de identidad, el principio de no contradicción y el principio del tercero excluido (3). El principio de identidad establece simple y llanamente que lo que es es (una rosa es una rosa). El principio de no contradicción, por su parte, establece que todo enunciado en el que se unan una frase y su negación mediante la conjunción copulativa "y", es falso (esta flor es una rosa y no es una rosa). Aristóteles dice al respecto que nada puede ser y no ser al mismo tiempo y en el mismo aspecto o, en la traducción de Calvo-Martínez que "es imposible que lo mismo se dé y no se dé en lo mismo a la vez y en el mismo sentido" (4). El principio del tercero excluido (en latín medieval tertium non datur: un tercero no se da), establece que al afirmar algo de un objeto sólo puede ser cierto uno de dos enunciados contradictorios, sin lugar para un enunciado intermedio. Con mayor frecuencia se enuncia diciendo que todo enunciado en el que se unan una frase y su negación mediante la conjunción disyuntiva "o", es verdadera (esta flor es una rosa o no es una rosa). Aristóteles dice al respecto que "tampoco puede darse un término intermedio entre los contradictorios, sino que necesariamente se ha de afirmar uno de ellos, sea el que sea, de una misma cosa" (5).

Sin lugar a dudas la frase de Stein es un claro ejemplo, en el campo de la lógica, del principio de identidad y, en el campo de la literatura, una alusión nada sutil a una frase que Shakespeare pone en boca de la protagonista de Romeo y Julieta en la segunda escena del segundo acto durante un encuentro clandestino que tiene por escenario el jardín de los Capuleto. Romeo Montesco (o Montagüe, como traduce el Marqués de Dos hermanas) ha saltado, como un ladrón, el muro del jardín de los Capuleto y espía hacia la ventana en la que, espera, aparecerá Julieta Capuleto. Efectivamente, Julieta se hace presente en el balcón y, dirigiéndose a las tinieblas del jardín sin saber que allí se encuentra oculto su amado Romeo, declama en voz alta: "[...] abjura tu nombre; o, si no quieres esto, jura solamente amarme y ceso de ser una Capuleto". Creyéndose a solas, Julieta continúa dirigiéndose a un imaginario Romeo, aunque los espectadores sabemos que, por una coincidencia propia del genio de Shakespeare, el Romeo de carne y hueso está allí en donde ella cree que sólo lo imagina: "Sólo tu nombre es mi enemigo. [Tú eres tú propio, no un Montagüe pues.] ¿Un Montagüe? ¿Qué es esto? Ni es piano, ni pie, ni brazo, ni rostro, ni otro [algún varonil] componente. [¡Oh! ¡Sé otro nombre cualquiera]” (6.) Las razones para que Julieta solicite a su amado que borre su nombre (o por las que ella quiere renunciar al suyo) son harto conocidas: en Verona la enemistad entre las familias Montesco y Capuleto no es palabrería inane; puede llegar a ser mortal como lo prueba el dramático final de la obra. Es en ese punto de la trama cuando, segura de que
Dr. Eugenio Matijasevic: Editor General Acta Médica Colombiana. Bogotá, D.C. (Colombia). E-mail: eugenio.matijasevic@gmail.com Recibido: 22/IV/2015 Aceptado: 23/IV/2015 
Romeo Montesco conservaría la tan querida perfección que posee aunque tuviese otro nombre exclama: "What's in a name? that which we call a rosel By any other name would smell as sweet;" (¿Qué hay en un nombre? Eso que llamamos rosa, con cualquier otro nombre aromaría igual de dulcemente) (7).

Los nombres de las especializaciones de la medicina son sorprendentes, nos dice William Bean, "algunos se refieren a distritos del cuerpo (oftalmología, dermatología), algunos a técnicas (radiología, cirugía), algunos a la edad (pediatría, geriatría) y algunos a imperativos territoriales (medicina preventiva, higiene industrial)" (8). Con "imperativos territoriales" Bean quiere enfatizar el hecho de que algunas especialidades médicas no poseen un campo específico de acción. La medicina preventiva y la higiene industrial, para continuar con sus ejemplos, no tendrían por qué ser coto exclusivo de ciertos especialistas sino que todos los médicos, pediatras, geriatras, oftalmólogos, ortopedistas, cirujanos y demás, tendrían que tener en su formación los elementos indispensables de cada una de esas disciplinas en su propia área. Aunque Bean no lo diga, el término imperativo territorial fue tomado prestado de un libro de Robert Ardrey publicado en 1966 y todavía famoso en la época en la que Bean escribió su artículo, llamado precisamente The Territorial Imperative. En él su autor describe muchas de las conductas humanas más cotidianas reduciéndolas a simples ejemplificaciones de su supuesta ley biológica del imperativo territorial: la necesidad que tienen los animales, entre ellos el animal humano, de reclamar para sí un territorio y defenderlo contra todos los miembros de su clase. El subtítulo del libro lo dice todo: A Personal Investigation into the Animal Origins of Property and Nations (una investigación personal al interior de los orígenes animales de la propiedad y las naciones) (9).

Pero entre los nombres de especialidades médicas, continúa Bean, el más sorprendente de todos, por lo confuso e irritante (annoying), es el de medicina interna. Para empezar, esta denominación no se refiere a ningún distrito del cuerpo (como la urología), tampoco se refiere a una técnica (como la radioterapia), tampoco a una edad (pues a pesar de los vanos intentos del American College of Physicians por crear en la conciencia pública identidad entre medicina interna y "medicina para adultos" el nombre de medicina interna, como tal, no dice nada con respecto a la edad) y, mucho menos, a un imperativo territorial.

Volviendo a la pregunta de Julieta ("¿Qué hay en un nombre?") y a su respuesta implícita ("nada”, puesto que la rosa aromaría igual con cualquier otro nombre), resulta tentador transformar su respuesta en un principio; no un principio de la lógica, pero sí un principio para la vida cotidiana que podríamos reformular diciendo: "en un nombre no hay nada, lo importante no es el nombre sino lo que hace aquel que es denominado con ese nombre, pues lo seguiría haciendo bajo cualquiera otra denominación". Ahora bien, si aplicamos el Principio Julieta (por darle algún nombre) a la extraña denominación de nuestra especialidad, habría que llegar a idéntica conclusión: si nuestra especialidad fuese llamada de otra manera, nosotros los internistas seguiríamos bajo otra denominación haciendo el mismo trabajo que hacemos, con idéntica calidad y con idéntico imperativo irrenunciable de dar el máximo en pro de nuestros pacientes. Aunque, pensándolo bien, sí habría algunas diferencias; no tendíamos que oír a la enfermera del servicio de urgencias diciéndonos antes de responder una interconsulta: "tiene que explicarle muy bien a la paciente quién es usted, doctor, porque cuando le dijimos que iba a venir a evaluarla el internista, insistió en que no se iba a dejar ver de ningún interno y exigió que la viera un especialista" (como si esa equivalencia, esa igualdad imaginada entre internista e interno, fuese una afrenta al principio de identidad); y no tendríamos que oír preguntas del calibre de "¿como así que usted se dedica a la medicina interna y trabaja en consulta externa?"(como si esa conjunción, esa " $y$ ", fuese una afrenta al principio de no contradicción) o " ¿y usted, siendo internista, por qué sabe tanto de la piel; ustedes estudian todo o estudian solamente los órganos internos?" (como si esa conjunción, esa "o", fuese una afrenta al principio del tercero excluido).

Sin ir en contravía del principio de identidad, pero acatando de plano el Principio Julieta, habría que insistir en que los internistas somos lo que somos pero, sobre todo, somos lo que hacemos. La mejor manera de definir un oficio es mostrando qué hace quien lo practica. Para el caso, no tiene ningún interés definir La Medicina Interna, así, con mayúsculas, sin haber mostrado primero qué hace un internista. Pero una definición ostensiva como la que propongo, que se lleva a cabo mostrando aquello que uno pretende definir (como cuando definimos qué es una rosa señalando una rosa en medio de otras flores), resulta demasiado lenta y engorrosa al tratar de definir a un internista, sobre todo porque los internistas hacemos demasiadas cosas: es posible encontrarlos en los servicios de urgencias, en las unidades de cuidado intensivo, en las áreas de hospitalización, en consulta externa, educando colegas en formación o futuros colegas, investigando, dando conferencias y, en fin, en innumerables e inverosímiles lugares: en las salas de hemodinamia de los departamentos de radiología, al pié de las máquinas de hemodiálisis de las unidades renales, junto a las gammacámaras de medicina nuclear, sosteniendo un endoscopio frente a un paciente bajo sedación o un fibrobroncoscopio frente a un paciente despierto, detrás de un microscopio en hematología o de un aparato de ultrasonido en cardiología o en reumatología y hasta escribiendo editoriales, para mencionar sólo unos cuantos. Y, puesto que la rosa con otro nombre aromaría igual, las personas que hacemos todo eso seguiríamos haciéndolo aunque no nos llamasen internistas.

De hecho, las personas que hacemos todo eso no hemos tenido siempre esa denominación. En Francia hicimos parte de un grupo más amplio, el de los médecins généralistes, hasta bien avanzado el siglo XX y sólo comenzaron a lla- 
marnos internistes cuando, en 1966, la Union Européenne des Médecins Spécialistes reconoció el papel de consultantes de los internistas trazando fronteras (bastante difusas, por cierto) entre la medicina interna, la medicina general y las otras especialidades médicas. En 1970 el Conseil National de l'Ordre des Médecins estableció los criterios de cualificación de los internistas y confirmó la necesidad de una formación hospitalaria polivalente, pero el diploma de certificación en medicina interna sólo fue creado en 1984 dando "registre d'état civil" a los internistas casi un siglo después que Alemania (10).

En Australia y Nueva Zelandia nos denominan physicians, denominación con la que es factible para el público en general diferenciarnos claramente de un general practitio$n e r$, pues se requiere un tiempo de entrenamiento adicional al entrenamiento universitario médico inicial, a veces hasta de ocho años, para que el Royal Australasian College of Physicians certifique a un médico como physician (11).

Esta tradición Australiana y Neozelandesa se remonta a la época en la que ambos países eran colonias del vasto Imperio Británico. De hecho, en la Gran Bretaña, un médico especializado en todo lo que nosotros los internistas colombianos sabemos hacer y estamos autorizados a hacer (todo ello, obviamente, después de un tiempo de entrenamiento mucho mayor que el de un general practitioner) recibe el nombre de physician. Sin embargo, en las últimas décadas, ha aparecido en los curricula de las facultades de medicina de Gran Bretaña la denominación General Internal Medicine para nuestra especialidad, seguramente mostrando una tendencia a homogeneizar los títulos con los demás países de la Comunidad Económica Europea y con Estados Unidos de Norteamérica y Canadá. Los especialistas en General Internal Medicine, por ahora, siguen siendo llamados physicians en Gran Bretaña, mientras que la generalidad de los angloparlantes británicos se resisten a utilizar la denominación internist.

En Gran Bretaña la formación de un physician requiere, después de la graduación como general practitioner, dos años de entrenamiento en un Foundation Programme idéntico para todas las subespecialidades médicas y, después de este período, debe realizar el entrenamiento específico de la subespecialización que haya elegido (cardiología, nefrología, endocrinología, etc.) en un core training programme. $\mathrm{Si}$ no se desea una subespecialidad, tiene la opción de continuar como especialista en general internal medicine, caso en el cual puede elegir entre dos posibles core training programmes: el Core Medical Training y el Acute Care Common Stem-Acute Medicine (12). En cualquiera de los dos casos los especialistas en general internal medicine tienen en la actualidad su carrera asegurada pues, de acuerdo con el Royal College of Physicians of London, el papel de los primeros (Core Medical Training) será cada vez más fuerte como consultantes en todo el National Health System de Gran Bretaña (13), y el de los segundos (Acute Care Common Stem) será el eje del cuidado en los hospitales del futuro (14).
En los Estados Unidos de América, a diferencia de Gran Bretaña y diversos países de la Commonwealth, se refieren a todo general practitioner, especializado o no, como physician mientras que al especialista en internal medicine le dan el título de internist. En efecto, la American Medical Association (AMA, creada en 1847) llama physician a cualquiera de sus miembros, independientemente de su especialidad o, incluso, si se trata de general practitioners (15). También la American Osteopathic Association (AOA, creada en 1897) denomina physicians a sus miembros pues todos los osteópatas graduados en los Estados Unidos de América, a diferencia de los osteópatas europeos, tienen licencia para ejercer como médicos, prescribir medicamentos y practicar intervenciones quirúrgicas pues su proceso educativo, además del entrenamiento en osteopathic manipulative treatment (tratamiento manipulativo osteopático), incluye entrenamiento completo en medicina (16).

La diferencia entre el uso del término physician en el inglés británico y en el inglés norteamericano no es extraña si tenemos presente que la historia de la especialización en ambos países ha sido bien diferente, aunque ahora tiendan a converger. A diferencia de la AMA y de la AOA, sin embargo, el American College of Physicians (ACP, creado en 1915), ha conservado para sus miembros la denominación de physicians en el antiguo y casi olvidado sentido del inglés británico. Sin embargo la diferencia entre un physician británico, australiano o neozelandés y un internist estadounidense es sólo cuestión de nombre pues, de hecho, de la misma manera que en Gran Bretaña la tendencia en las últimas décadas es a que los physicians, los especialistas en General Internal Medicine, trabajen en los hospitales manejando los pacientes desde el ingreso como parte del Acute Care Common Stem, la tendencia en los Estados Unidos de América es a que los internists, los especialistas en General Internal Medicine, trabajen como hospitalists, denominación introducida hacia 1996 para referirse a los especialistas que trabajan en los hospitales manejando los pacientes desde el ingreso (17). En Canadá, a pesar de su historia como antigua colonia británica, se mantienen denominaciones más afines con las de Estados Unidos de América para los especialistas en medicina interna (internist en el Canadá anglófono e interniste en el francófono) (18), a pesar de la estrecha relación (que podríamos denominar filial) entre la Canadian Society of Internal Medicine o Société Canadiense de Médecine Interne con el ACP (19).

Paul Bruce Beeson, quien acuñó el concepto de fever of unexplained origin (fiebre de origen desconocido) (20), siendo ya Emeritus Professor de la Universidad de Washington en Seattle, afirmaba en 1986 que prefería el término physician británico al término norteamericano internist (21).

¿Por qué terminamos llamándonos internistas?. El empleo de este nombre en Colombia y en los países iberoamericanos proviene directamente del inglés internists derivado de internal medicine, sin lugar a dudas porque la mayoría de los primeros especialistas en el campo se 
formaron en los Estados Unidos de América, aunque cabe recordar que José María Lombana Barreneche, nuestro padre, se formó como internista en Ambalema, con mucho estudio y práctica continua, sin haber ido ni a los Estados Unidos ni a Francia que era, para su época, la Meca de los médicos latinoamericanos que deseaban complementar su formación en el exterior.

Sin embargo, el nacimiento oficial de la medicina interna como disciplina autónoma y con ese nombre no tuvo lugar en los Estados Unidos de América sino que, seguramente, ocurrió entre el 20 y el 22 de abril de 1882 cuando en Wiesbaden tuvo lugar el primer congreso de medicina interna cuyas Verhandlungen des Kongresses für Innere Medizin (Actas del Congreso de Medicina Interna) algunos afortunados germanoparlantes pueden leer ahora en internet (22) y deleitarse, por ejemplo, con trabajos tan originales como una ponencia de Robert Koch Ueber die Aetiologie der Tuberculose (Sobre la Etiología de la Tuberculosis) o un trabajo de Friedrich Theodor von Frerichs sobre Pathologie des Morbos Brightii (Patología de la Enfermedad de Bright, es decir, de la insuficiencia renal crónica) u otro de Winkler Ueber den Stoffwechsel im Fieber (Sobre el Metabolismo en la Fiebre)... realmente tuvieron que ser tres días memorables.

Es gracias a este congreso inaugural, se ha dicho siempre, que la expresión medicina interna se originó en Alemania y llegó hasta nosotros a través de la expresión inglesa internal medicine, derivada a su vez del alemán Innere Medizin. Incluso hay quienes llevan la leyenda más allá y afirman que fue Frerichs quien acuñó el término Innere Medizin durante el congreso Wiesbaden. Se olvida, sin embargo que si en 1882 hubo un primer congreso de medicina interna en Alemania, dedicado a los avances de una rama de la medicina con ese nombre, es porque la especialidad hacía un buen tiempo que se practicaba y existía un buen número de médicos que se consideraban a sí mismos expertos en ese campo, los suficientes como para que fuese factible la organización de un congreso de esa naturaleza. Sin lugar a dudas para entonces la especialidad ya estaba constituida o comenzando a constituirse con ese nombre y el término Innere Medizin se utilizaba desde antes del congreso (23).

En efecto, el primer uso documentado de la expresión medicina interna o, en este caso, medicina interna, así, en italiano, data del año 1839, con ocasión del primer Congresso degli Scienziati Italiani (congreso de los científicos italianos), celebrado en Pisa. Al parecer el término era empleado con el fin de diferenciar una cierta forma de ejercer la medicina de la medicina esterna, que correspondía en aquel entonces al ámbito de la cirugía (24). Pero ambas expresiones italianas, medicina interna y medicina esterna, tenían claros antecedentes en la medicina francesa de unas décadas previas.

La historia se remonta a la Revolución Francesa y ha sido contaba con precisión por Sicard (25): "al comenzar el siglo XVIII -cito a Sicard- el cirujano no era más que un barbero, pero al finalizar el siglo se había transformado en un anatomista, un clínico, un patólogo". El 2 de marzo de 1791 -continúo citando a Sicard-, en plena Revolución Francesa, la Asamblea Nacional (que dirigió los destinos de Francia de manera transitoria del 9 de junio de 1789 al 17 de julio del mismo año) suprimió la Corporation des Chirurgiens (corporación de cirujanos) y proclamó la libertad de ejercer la medicina y la cirugía sin condiciones legales de estudios, de grados y de diplomas. Esto dio lugar a increíbles abusos y por ello la Convención Nacional (que detentó el poder legislativo de Francia, y en parte el ejecutivo, desde el 20 de septiembre de 1792 al 26 de octubre de 1795) creó tres escuelas de medicina, una en París, otra en Montpellier y la tercera en Estrasburgo. La de París tomó posesión de inmediato del edificio de la Academia de Cirugía en donde se estableció, dando lugar a una creciente rivalidad entre médicos y cirujanos, rivalidad que fue zanjada al final del siglo por la Convención Nacional al aceptar que la medicina y la cirugía eran dos partes de la misma ciencia. En 1802 -sigo con Sicard-, Dominique Larrey le solicitó a Napoleón Bonaparte que restableciera la Academia de Cirugía y le diera independencia a los cirujanos, pero éste, siguiendo el consejo de Chaptal, se rehusó y, en 1804, ante la renovación de la solicitud, Bonaparte rechazó definitivamente el proyecto y decretó que la Société de l'Ecole de Médecine (Sociedad de la Facultad de Medicina) de París acogiera a los cirujanos.

Es por ello que para 1813 la enseñanza de la medicina en la Facultad de Medicina de París estaba dividida en una Clinique Externe, de la que se encargaban Pelletan y Boyer, una Clinique Interne a cargo de Corvisart y Leroux y una Clinique de Perfectionnement que se llevaba a cabo en el Hospice de Perfectionnement (Hospicio de Perfeccionamiento, un claro antecesor del Hospital Universitario) de la facultad de medicina, bajo la dirección de Antoine Dubois y Petit-Radel (26).

En la Clinique Externe se enseñaban, mediante conferencias magistrales, las alteraciones de la salud susceptibles de ser tratadas mediante métodos quirúrgicos. El nombre no fue producto del azar: dado el escaso desarrollo de la cirugía de la época [faltaban aún tres décadas para el descubrimiento la anestesia (27) y más de cinco para que Lister pusiera a punto la antisepsia (28)] los procedimientos quirúrgicos tenían limitadas sus posibilidades de acción terapéutica a las lesiones superficiales accesibles a su incipiente tecnología, sin posibilidad alguna de acceso a las lesiones localizadas en las cavidades internas del cuerpo; de allí la denominación de Clinique Externe a esta etapa de la educación médica que en los textos escritos por los expertos tomaba el nombre de Pathologie Externe.

En la Clinique Interne se enseñaban, mediante conferencias magistrales, aquellas alteraciones de la salud que, por su carácter sistémico o por estar localizadas en las cavidades profundas del cuerpo, resultaban inaccesibles desde el exterior a la técnica quirúrgica de la época. Una 
larga tradición en las facultades de medicina de Francia hizo que muchos médicos de todas partes de Europa e incluso de América fueran a oír las conferencias de Clinique Interne de connotados Professeurs de cuyos nombres aún tenemos noticia en muchos de los epónimos que utilizamos; fueron famosos, y existen recuentos de sus clases, los cursos de Pathologie Interne dictados por François Broussais en 1819 y por Gabriel Andral en 1833 (29).

La influencia francesa, tanto en Clinique Interne como en Clinique Externe se extendió sobre todo a Alemania, Italia y también a Iberoamérica. Joacim Cil Borés, catedrático de la Facultad de Medicina de Barcelona, tenía muy claro en 1852 que "medicina externa" y "patología quirúrgica" eran una y la misma cosa y escribió al comienzo de la minuta del curso que sobre el tema dictaba en la facultad: "Tratado de afectos o enfermedades externas, medicina externa o cirugía, y también patología quirúrgica, se ha llamado aquella parte de la ciencia médica que enseña a conocer y tratar los afectos externos" (30).

Muchos de nuestros médicos del siglo XIX se formaron en Francia (Antonio Vargas Reyes, Andrés María Pardo, Carlos Esguerra Gaitán, Carlos Putnam Tanco, Evaristo García, Hipólito Machado, Julián Escobar Fernández, Manuel Plata Azuero, Nicolás Osorio, Pío Rengifo, Proto Gómez, Ricardo Rodríguez Roldán, Tomas Quevedo Restrepo, para nombrar sólo unos cuantos) (31). Con ellos trajeron a Colombia las mismas controversias que en Francia y Europa avivaban continuamente el desarrollo teórico de la medicina de la época a partir de tradiciones diversas que tenían su epicentro en una región o en una facultad de medicina o a veces en un personaje: las tradiciones anatomoclínica, fisiopatológica, etiológica e incluso las mecanicista y vitalista (32).

A comienzos del siglo XIX el influjo francés sobre la medicina de los pueblos germanoparlantes de Europa Central (que pronto se unirían en la Confederación Germánica, por un lado, y el imperio de Austria por el otro, y que más tarde se transformarían en el Imperio Alemán y el Imperio Austrohúngaro, respectivamente), hizo que, de la misma manera que en Italia se hablaba de medicina esterna y medicina interna, en alemán se hiciera referencia a la Äußere Medizin y la Innere Medizin para referirse, respectivamente, a la Clinique Externe y a la Clinique Interne (33) al igual que surgieron términos como Äußere Krankheiten (enfermedades externas) e Innere Krankheiten (enfermedades internas) para referirse a la Pathologie Externe y a la Pathologie Interne, respectivamente.

Pero con el paso del tiempo y con los cambios económicos arrastrados por las últimas décadas de la primera revolución industrial, se dieron cambios en el mapa geopolítico de Europa que modificaron de manera implacable los polos de atracción para científicos y estudiantes de las más diversas ciencias. En consecuencia, la preeminencia de Francia en el desarrollo de la medicina fue dando paso a un aumento progresivo del influjo de las escuelas de me- dicina centro-europeas de lengua alemana sobre todas las demás. Esta era la situación en las décadas finales del siglo XIX, cuando la mayoría de los médicos norteamericanos que deseaban ponerse al día en técnicas y desarrollos científicos en su campo ya no iban a Francia a aprender sino que hacían la peregrinación por las escuelas de medicina del Imperio Alemán y del Imperio Austrohúngaro. Era la época, además, en la que el desarrollo de la química, de la microscopía, de la patología, de las técnicas de tinción en patología, de la fisiología, de la bacteriología, se habían desarrollado lo suficiente para poder salir de los laboratorios de investigación de los centros académicos y comenzar a ser utilizados por los médicos a la cabecera del paciente. Se empezaron a formar, entonces, médicos que venían desde la tradición de la Clinique Interne y de la Pathologie Interne, que tenían a su disposición para el estudio de las enfermedades en sus pacientes instrumentos, dispositivos e instituciones nuevos, como el examen clínico (que desde hacía poco había sumado a la inspección y a la palpación, las sempiternas técnicas de exploración, la percusión de Joseph Leopold Auenbrugger y la auscultación mediata de René Théophile Hyacinthe Laënnec), y los laboratorios de química, de fisiología, de microbiología y de patología. Pero ante todo, estos nuevos médicos disponían de un método de pensamiento clínico basado en el método pergeñado por Galileo, Descartes, Bacon y Newton (el método científico) que permitía separar claramente el conocimiento aportado por la ciencia de las creencias provenientes de la fe, las órdenes emanadas de la autoridad y las tradiciones recibidas con las costumbres. Estos nuevos médicos, especialistas en Innere Medizin, ejercían la profesión de Hipócrates con nuevos instrumentos pero habían retornado al antiguo postulado que hizo poderosa la escuela de Cos: todas las enfermedades son susceptibles de ser estudiadas hasta descubrir su origen, y no existen ni morbus divinus ni morbus sacer producidos por los dioses, sólo existen causas naturales. Innere había adquirido en este caso un nuevo sentido, mucho más

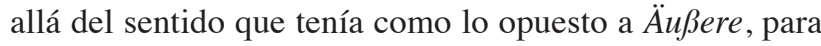
transformarse en la capacidad del médico para ir, no ya al interior del cuerpo del paciente, sino al interior del problema clínico del paciente "utilizando el conocimiento obtenido mediante las ciencias experimentales para llegar a la base de los signos y síntomas de una enfermedad, utilizando el método científico en lugar del dogma" (34).

Después de haber culminado sus años de medicina básica en la McGill University Faculty of Medicine y después de haber estudiado fisiología en Londres entre 1872 y 1873, el joven William Osler (tenía 24 años) se dirigió, ¡cómo no!, a Berlín y a Viena en donde, entre 1873 y 1874, realizó varias pasantías con los mejores especialistas de la época. En Berlín estudió con Rudolf Virchow (padre de la teoría celular y padre de la patología) y con los dos más eminentes clínicos de entonces: Ludwig Traube y Friedrich Theodor von Frerichs (quien habría de dirigir 9 años más tarde el Primer Congreso de Medicina Interna de que se 
tenga noticia). En Viena estudió pediatría con Hermann Wiederhoffer, dermatología con Ferdinand Hebra, medicina general con Heinrich von Bamberger y enfermedades del oído con Adam Politzer (35). Para contar el resto de su historia basta con describir la ascensión de un meteoro: regresó a la Universidad de McGill como profesor en 1874, fue nombrado Jefe de Medicina Clínica de la Universidad de Pensilvania en Filadelfia en 1884, aceptó el cargo de primer Physician-in-Chief del recién creado Johns Hopkins Hospital en Baltimore en 1889 (en donde cuatro años más tarde ayudaría a crear la Johns Hopkins University School of Medicine) y fue nombrado Regius Chair of Medicine en la Universidad de Oxford en 1905. Pero en el intervalo, en 1885 , fue uno de los siete miembros fundadores de la Association of American Physicians (AAP) de la que sería presidente en 1895. Con motivo de la décima reunión de la AAP, Osler se encargó, como correspondía, de la alocución presidencial, a la que dio el título de Internal Medicine as a Vocation (36). Es esta la primera mención documentada del término Internal Medicine en los Estados Unidos de América (37).

La capacidad de Osler como clínico sigue siendo paradigmática, al igual que se le recuerda por su disciplina e inagotable capacidad de trabajo y por su capacidad organizativa tanto desde el punto de vista médico como administrativo; pero él en realidad quería ser recordado por su contribución, a veces soslayada, a la educación médica. En primer lugar trasladó la enseñanza de la medicina de los salones de conferencias a la cabecera de los pacientes e impulsó la idea de una educación médica basada en menos clases magistrales y más bedside teaching rounds, rondas académico-asistenciales realizadas directamente en los servicios de medicina interna con la presencia de gran parte del equipo médico del departamento, incluyendo profesores, residentes, internos, alumnos (a partir del tercer año estaban autorizados para realizar la anamnesis, el examen físico e incluso exámenes básicos de laboratorio como el hemograma, el uroanálisis, el coprológico y la tinción Gram de diversas secreciones), enfermeras del servicio, terapeutas, etc. En segundo lugar, creó el programa de residencia médica, en el cual el residente, a más de estar recibiendo formación de postgrado en el área de la medicina que le interesa, hace parte del equipo médico que atiende de manera directa a los pacientes y está, por tanto, adscrito no sólo a la facultad de medicina como estudiante de postgrado sino también al hospital como trabajador en entrenamiento, una idea que rápidamente se diseminó a todas las facultades de medicina de los Estados Unidos y, más tarde, a muchos otros países del mundo (38) con la notable excepción de Colombia.

En 1908 Abraham Flexner ingresó como research scholar en la Carnegie Foundation for the Advancement of Teaching y dos años más tarde publicó su famoso "Informe", después de visitar las 155 facultades de medicina de los Estados Unidos de América y Canadá y de examinar en ellas, a lo largo de 18 meses, cinco áreas que incluían: requisitos de ingreso, número y entrenamiento de los profesores, dotaciones y costo de matrícula, calidad de los laboratorios y disponibilidad de un hospital universitario en donde médicos y cirujanos sirvieran como profesores clínicos (39). Lo que encontró Flexner no era muy diferente a lo que Osler estaba cambiando en Johns Hopkins: "Cada día -dice Flexner en su Informe- los estudiantes son sometidos a conferencias y recitaciones interminables. Después de una larga mañana dedicados a la disección o a una serie de secciones de preguntas, podrán sentarse en la tarde, agotados, a escuchar tres o cuatro o incluso cinco conferencias dictadas de manera metódica por profesores de tiempo parcial [...] En las noches se les deja libres para leer y preparar las recitaciones. Si son lo suficientemente afortunados para conseguir entrar a un hospital, observan más que participan" (40). La posición de Flexner desde el punto de vista educativo ( debemos recordar que era educador -o pedagogo, como se prefiere decir ahora-, no médico?) era bastante clara: "Una educación en Medicina debe incluir tanto aprender como aprender cómo. El estudiante no puede saber a menos que sepa cómo". Su postura desde punto de vista sociopolítico está también clara, y fuerte: "El Médico es un instrumento de la sociedad y su función se está volviendo social y preventiva más que individual y curativa". En la primera, la posición pedagógica, a pesar de la animada controversia que mantuvieron después, Flexner y Osler estaban en el mismo campo, de hecho para Flexner una facultad de medicina como la que Osler había ayudado a crear en la Universidad Johns Hopkins fue a la vez una revelación y un modelo para la evaluación de las demás. Después del informe Flexner todas las facultades quisieron ser como la de Johns Hopkins (o desaparecieron: entre 1910 y 1920 el número de facultades de medicina en Norteamérica disminuyó de 131 a 81 y el número de estudiantes cayó de 4400 a 2529) (41), adoptando en este sentido una educación de tipo Osleriano. Flexner, sin embargo, iba más allá y quería que la investigación y el laboratorio y la industria estuvieran directamente involucrados en la educación en la universidad, a lo que Osler se opuso siempre pues lo veía como una intromisión indeseable en la relación medico-paciente (42).

A partir de ese punto de inflexión en la educación médica en Norteamérica, todo cambió y las facultades de medicina de los Estados Unidos se convirtieron en la Meca para los estudiantes latinoamericanos e incluso, después de la segunda guerra mundial, también para muchos europeos.

El resto es ya la historia de la Medicina Interna en Colombia, que ha sido muy bien contada en un libro que a todos recomiendo, publicado por la Asociación Colombiana de Medicina Interna y que se llama, precisamente, La Medicina Interna en Colombia (43). No tiene sentido repetir lo que allí está contado (y muy bien contado) por los protagonistas de nuestra historia. El lector avezado encontrará en él desde la historia de cuatro figuras señeras de la medicina interna de nuestro país (44) hasta análisis 
sesudos sobre el presente (45) y el futuro (46) de nuestra medicina interna, pasando por el valor de la medicina interna para la formación de nuestros médicos (47), el papel de la investigación científica en dicha formación (48) y el papel de la información médica en la sociedad (49), para llegar, al final, a una historia de esta revista sobre la que no voy a detenerme porque allí está perfectamente bien contada (50) y aparecen en ella personajes que, mirados desde aquí, desde ahora, desde lo que en ese entonces era el futuro, parecen ya personajes de leyenda: Fernando Chalem, Jorge Escandón, Roso Alfredo Cala, Ernesto Laverde, Jimeno Ramírez... me detengo incapaz de evocar tantos héroes al unísono; para los antiguos griegos la epifanía de un dios o de ciertos héroes estaba más allá de lo que un simple mortal podía soportar sin que su cuerpo ardiese en llamas de manera espontánea.

Acta Médica Colombiana cumple en este año 40 años de publicación ininterrumpida, 40 años publicando lo que sabemos hacer los internistas en Colombia, publicando lo que nos hace ser lo que somos independientemente del nombre que nos den. Cuarenta años puede no parecer demasiado tiempo si se los compara a nivel mundial con la larga vida de otras publicaciones biomédicas como JAMA, fundada en 1883; The Lancet, fundada en 1823 o The New England Journal of Medicine fundada en 1812. Ni qué decir si la comparamos con otras publicaciones científicas periódicas arbitradas por pares, la primera de las cuales, Philosophical Transactions of the Royal Society, fundada por Henry Oldenburg en 1665, cumple este año 350 años de publicación ininterrumpida. Pero es nuestra revista y varias generaciones de médicos colombianos hemos crecido a su sombra.

Al final de El Nombre de la Rosa (51), cuyo título, todos apostábamos, tendría que ver con la rosa de la Julieta de Shakespeare, viene uno a enterarse que no, que se trata de otra rosa, de una rosa medieval como correspondía al buen medievalista que es Umberto Eco, una rosa tomada de un poema de Bernardo Morliacense sobre el tema de ubi sunt (¿dónde están?), muy en el sentido en el que unos siglos más tarde Don Jorge Manrique, se preguntaría en las coplas a la muerte del Maese Don Rodrigo su padre: “¿Qué se hizo el rey don Juan?/ Los infantes de Aragón/ ¿qué se hizieron?/ ¿Qué fue de tanto galán,/ qué de tanta invención/ qué trajeron?/¿Fueron sino devaneos,/ qué fueron sino verduras/ de las eras,/ las justas y los torneos,/ paramentos, bordaduras/ y cimeras?" (52). La rosa de Eco y Bernardo Morliacense no es una rosa como la de Julieta, como aquella rosa que, incluso bajo otro nombre, aromaría igual, sino una rosa ausente, ida, como los galanes e invenciones de Manrique: stat rosa pristina nomine, nomina nuda tenemus (la rosa, que era, [ahora] sólo existe en el nombre, sólo nos queda su nombre desnudo). Indudablemente existe una clara contradicción entre la rosa de Shakespeare y la de Eco: mientras que para el primero las rosas, las personas y eventos del mundo siguen siendo igual de amables incluso bajo otro nombre, para el segundo las rosas, las personas y eventos del mundo desaparecen y sólo nos queda su nombre. Shakespeare es un realista a la manera Aristotélica, que acepta la existencia de universales abstractos (rosa, internista) instanciados en objetos concretos (esta rosa, Roso Alfredo Cala), mientras que Eco (o por lo menos la rosa de Eco) es un nominalista para quien los universales abstractos no existen, sólo son nombres en nuestra mente, y perdurarán en ella incluso cuando los objetos (personas y eventos del mundo) de los cuales enunciaban propiedades hayan desaparecido.

¿Qué hay en un nombre? ¿Qué ocurrirá con nosotros los internistas -los seres humanos concretos- ahora que es posible que en Estados Unidos de América cambiemos de nombre por hospitalists o que en Gran Bretaña cambiemos de nombre por especialistas en Acute-care? ¿Qué pasará con la denominación de la medicina interna -así, en abstracto- ahora que los especialistas en medicina interna hacemos tantas otras cosas que la medicina interna general es cosa del pasado?. Yo, de todas maneras, me decanto por la rosa de Julieta: cualquiera que sea el nombre con el que nos designen o el nombre que le demos a nuestra especialidad, no debemos olvidar un sólo instante que desde hace 40 años honramos en la práctica la definición que, en el primer Editorial publicado en esta revista, diera Roso Alfredo Cala de lo que es un internista: "El Internista debe ser el eje alrededor del cual se movilice todo el equipo de salud frente a un paciente. Es el asesor y consultante para otros especialistas; es el hombre que dirige y centraliza la acción médica, evitando la atomización del cuidado del enfermo" (53) .

\section{Referencias}

1. Cano JM (1991)."Una rosa es una rosa”[Grabado por Torroja A con la banda Mecano]. En "Aidalai”[Audio CD].BMG Music Spain (2005).

2. Stein G.Sacred Emily. En: Geography and Plays. Toronto: General Publishing Company; 1999: pp 178-188.

3. Béziau JY. What is the principle of identity (Identity, logic and congruence) En: Sautter FT, de Araujo Feitosa H (eds) Lógica: teoria, aplicações e reflexões. Campinas: Centro de Lógica, Epistemologia e História da Cie^ncia; 2004: pp.163-172.

4. Aristóteles. Metafísica. IV, 3, 1005b, 18-19. Calvo-Martínez T (Traductor) Madrid: Editorial Gredos; 1994: p 115.

5. Aristóteles. Metafísica. IV, 3, 1011b, 23-24. Calvo-Martínez T (Traductor) Madrid: Editorial Gredos; 1994: p 140.

6. Shakespeare W. Julieta y Romeo. En: Obras de William Shakespeare traducidas fielmente del original inglés por Matías de Velasco y Rojas, Marqués de Dos Hermanas. Volumen III. Madrid: R. Berenguillo: 1872. Consultado el 21 de febrero de 2015. Disponible en http://www.cervantesvirtual.com/servlet/SirveObras/01593074980144858540035/p0000001.htm

7. Shakespeare W. Romeo and Juliet (Act II, Scene II, verses 47-48). En: The Oxford's Shakespeare: the complete works of William Shakespeare.London: Oxford University Press; 1914. Consultado el 21 de febrero de 2015. Disponible en http://www.bartleby.com/70/3801.html

8. Bean WB. Origin of the Term Internal Medicine.N Engl J Med 1982; 306:182-183.

9. Ardrey R. The Territorial Imperative: A Personal Investigation into the Animal Origins of Property and Nations. New York: StoryDesign Ltd; 2014. Edición en formato digital: archivo Kindle.

10. Blanchardière A, Le Dinh T. Histoire de la médecine interne. Rev Med Interne1994; 15: 690-693.

11. The Royal Australasian College of Physicians. What is a Physician? [Internet]. Consultado el 21 de febrero de 2015. Disponible en:http:// 
web.archive.org/web/20080306053048/http://www.racp.edu.au/index. cfm?objectid=49EF1EB5-2A57-5487-D74DBAFBAE9143A3.

12. Joint Royal Colleges of Physicians Training Board. General Internal Medicine: About the specialty [Internet]. Consultado el 21 de febrero de 2015. Disponible en: http://www.jrcptb.org.uk/specialties/general-internal-medicine-gim.

13. Royal College of Physicians. Consultant physicians working with patients, revised 5th edition (online update). London: RCP, 2013, last updated 2015. Consultado el 21 de febrero de 2015. Disponible en: https://www.rcplondon.ac.uk/resources/ consultant-physicians-working-patients-0.

14. Future Hospital Commission. Future Hospital: caring for medical patients. A report from the Future Hospital Commission to the Royal College of Physicians [Internet]. Consultado el 21 de febrero de 2015. Disponible en: https://www. rcplondon.ac.uk/projects/future-hospital-commission.

15. American Medical Association. AMA Resources for Physicians [Internet]. Consultado el 21 de febrero de 2015. Disponible en: http://www.ama-assn.org/ ama/pub/physicians/physicians.page? .

16. American Osteopathic Association. About Osteopathic Medicine [Internet]. Consultado el 21 de febrero de 2015. Disponible en:http://www.osteopathic.org/ osteopathic-health/about-dos/about-osteopathic-medicine/Pages/default.aspx.

17. Wachter R, Goldman L. The emerging role of "hospitalists" in the American health care system. N Engl J Med 1996; 335 (7): 514-517.

18. Association des spécialistes en médecine interne du Québec (ASMIQ). Qu'estce qu'un interniste? [Internet]. Consultado el 21 de febrero de 2015. Disponible en: http://asmiq.org/asmiq-accueil/quest-ce-quun-interniste/.

19. Canadian Society of Internal Medicine. History of CSIM [Internet]. COnsultado el 21 de febrro de 2015. Disponibe en: http://www.csim.ca/fr/a-propos-ducsim/histoire-de-la-scmi.

20. Petersdorf RG, Beeson PB. Fever of unexplained origin: report on 100 cases. Medicine (Baltimore) 1961; 40: 1-30.

21. Beeson PB. One hundred years of American internal medicine. A view from the inside. Ann Intern Med 1986; 105(3): 436-444.

22. Leyden E, Seitz E. Verhandlungen des Kongresses für Innere Medizin. Wiesbaden:Verlag Von JF Bergmann; 1882. Consultado el 21 de febrero de 2015. Disponible en: https://archive.org/stream/verhandlungen01medigoog\#page/n4/ mode/2up.

23. Murillo-Godínez G. Algunos comentarios sobre la Historia de la Medicina Interna en México y en el mundo. Med Int Méx 2013; 29: 622-624

24. Federspil G. Presentazione dell'edizione italiana. En: Braunwald E, Fauci AS, Kasper DL (Editori) Harrison's Principi di Medicina Interna. Quindicesima edizione. Milano: McGraw Hill Italia; 2004 [Internet]. Consultado el 21 de febrero de 2015. Disponible en: http://www.ateneonline.it/pdf/indice_pref/2970-6_pres. pdf.

25. Sicard A. La chirurgie française au XVIIIe siècle. Histoire des sciences médicales 1994; 26 (2): 93-99.

26. Huard P, Imbault-Huart MJ. Structure et fonctionnement de la Facultéde Médecine de Paris en 1813. Revue d`histoire des sciences 1975; 28 (2): 139-168.

27. Morton WTG. Remarks on the proper mode of administering sulphuric ether by inhalation. Boston: Dutton and Wentworth printers; 1847 [Internet]. Consultado el 21 de febrero de 2015. Disponible en: https://archive.org/stream/39002011123958. med.yale.edu\#page/n5/mode/2up.

28. Lister J. On the antiseptic principle in the practice of surgery. The Lancet1867; 90, (2299): 353-356 [Internet], Consultado el 21 de febrero de 2015. Disponible en: http://www.sciencedirect.com/science/article/pii/S0140673602518274?np=y.

29. Rodríguez-Erdmann F. Origin of the term 'Internal Medicine'. Arch Intern Med $1983 ; 143$ (1): 184

30. Pera C. El Cuerpo Herido: Un Diccionario Filosófico de la Cirugía. Barcelona: Editions Universitat de Barcelona; 1999: p 177.

31. Quevedo E, Pérexz GE, Miranda N et al. Historia de la Medicina en Colombia.
Tomo III: Hacia una Profesión Liberal. Bogotá: Editorial Norma; 2010: pp 288289.

32. De Francisco A. La Medicina Interna en Bogotáa Mediados del siglo XX. En: Hernández CA, Ardila E, Niño MN (Eds). La Medicina Interna en Colombia. Bogotá: ACMI; 2006: pp 25-30.

33. Eckart WU. Illustrierte Geschichte der Medizin: Von der französischen Revolution bis zur Gegenwart. Berlin: Springer-Verlag; 2011: p 39.

34. Fordtran JS, Armstrong M, Emmett M, et al. The history of internal medicine at Baylor UniversityMedical Center, part 1. Baylor University Medical Center Proceedings 2004; 17: 9-22.

35. Bliss M. William Osler: A Life in Medicine. Toronto: University of Toronto Press; 1999: pp 76-77

36. Osler W. Internal medicine as a vocation. In: Aequanimitas with OtherAddresses to Medical Students, Nurses, and Practitioners of Medicine. Second Edition. London: HK Lewis; 1914: pp 137-152.

37. Fordtran JS, Armstrong M, Emmett M, et al. The history of internal medicine at Baylor University Medical Center, part 1. Baylor University Medical Center Proceedings 2004; 17: 9-22.

38. Johns Hopkins Medicine. The Four Founding Physicians [Internet]. Consultado el 21 de febrero de 2015. Disponible en:http://www.hopkinsmedicine.org/about/ history/history5.html.

39. Beck AH. The Flexner Report and the Standardization of American Medical Education. JAMA 2004; 291(17): 2139-2140.

40. Wear D. Iconoclast: Abraham Flexner and a Life in Learning. N Engl J Med 2002, 347: 2088-2089.

41. Tauber AI. The two faces of medical education: Flexner and Osler revisited Journal of the Royal Society of Medicine 1992; 85: 598-602.

42. Tauber AI. Op. Cit.

43. Hernández CA, Ardila E, Niño MN. La Medicina Interna en Colombia. Bogotá: ACMI; 2006.

44. Sarasti H. Cuatro figuras en la historia de la medicina colombiana y una mirada a sus orígenes. En: Hernández CA, Ardila E, Niño MN (Eds.). La Medicina Interna en Colombia. Bogotá: ACMI; 2006: pp 19-30.

45. Molina DI. Sistema de seguridad social en salud y ubicación laboral de los médicos internistas en Colombia. En: Hernández CA, Ardila E, Niño MN (Eds.) La Medicina Interna en Colombia. Bogotá: ACMI; 2006: pp 46-70.

46. D'Achiardi R. Futuro del internista en Colombia. En: Hernández CA, Ardila E, Niño MN (Eds.). La Medicina Interna en Colombia. Bogotá: ACMI; 2006: pp 71-81.

47. Maldonado D. Proyección de la medicina interna en la formación del medico. En: Hernández CA, Ardila E, Niño MN (Eds.). La Medicina Interna en Colombia Bogotá: ACMI; 2006: pp 82-96.

48. Gamarra G. La investigación en medicina interna en Colombia. En: Hernández CA, Ardila E, Niño MN (Eds.). La Medicina Interna en Colombia. Bogotá: ACMI; 2006: pp 97-105.

49. Ardila E. La medicina y los medios de comunicación. En: Hernández CA, Ardila E, Niño MN (Eds.). La Medicina Interna en Colombia. Bogotá: ACMI; 2006: pp 106-116.

50. Archila PE. Publicaciones de la ACMI: los oficios de Acta Médica Colombiana En: Hernández CA, Ardila E, Niño MN (Eds.). La Medicina Interna en Colombia. Bogotá: ACMI; 2006: pp 183-191.

51. Eco U. El Nombre de la Rosa. Apostillas al Nombre de la Rosa. Pochtar R (Traductor). Barcelona: Editorial Lumen; 2005. Edición en formato digital: archivo Ibooks.

52. Manrique J. Coplas que hizo Don Jorge Manrique a la muerte del Maestre de Santiago Don Rodrigo Manrique su padre. En: Jorge Manrique Poesía. Beltrán V (Editor). Barcelona: Editorial Crítica; 1993: pp 158-159.

53. Cala RA. Medicina Interna. Acta Med Colomb 1976; 1 (1): 1-2. 